\title{
Every patient should be enabled to stop the line
}

\author{
Sigall K Bell, ${ }^{1}$ William Martinez ${ }^{2}$
}

${ }^{1}$ Department of Medicine, Beth Israel Deaconess Medical Center, Harvard Medical School, Boston, Massachusetts, USA ${ }^{2}$ Department of Medicine, Vanderbilt University Medical Center, Nashville, Tennessee, USA

\section{Correspondence to}

Dr Sigall K Bell, Department of Medicine, Beth Israel Deaconess Medical Center, Harvard Medical School, Boston, MA 02215, USA; sbell1@bidmc.harvard.edu

Accepted 30 October 2018 Published Online First 22 November 2018

\section{Sinked}

- http://dx.doi.org/10.1136/ bmjqs-2018-008159

\section{Check for updates}

(C) Author(s) (or their employer(s)) 2019. No commercial re-use. See rights and permissions. Published by BMJ.

To cite: Bell SK, Martinez W. BMJ Qual Saf

2019;28:172-176.
When Taiichi Ohno introduced 'Stop the Line' manufacturing, people were sceptical. ${ }^{1}{ }^{2}$ Each assembly line worker was entrusted with the responsibility to push a red button to stop the line if she/ he noticed something wrong. The idea was to catch problems early, before they got out of control. But the approach seemed at odds with production goals, namely keeping assembly lines running at full speed. Why not have managers, more trained in production, oversee the line and make fewer stops? Ohno's idea seemed too risky to some managers, who resisted. Indeed, managers who implemented Stop the Line experienced a productivity drop. Investigating and fixing problems took time. But soon, things flipped. The teams using Stop the Line were faster and more reliable than those that did not and Stop the Line manufacturing became a standard Toyota approach.

When patients enter the hospital, they entrust clinicians to push a red button if they sense something wrong. But patients themselves, increasingly championed as 'members of the team' and 'co-producers' of health, are not always given a button nor taught how to use it. ${ }^{3-5}$ Patients and families-vigilant stakeholders-hold unique knowledge and can make important contributions to patient safety, having repeatedly demonstrated the ability to identify problems in care, including ones missed by clinicians. ${ }^{6-11}$ Parents, like James Titcombe, whose son died 9 days after birth from a delayed sepsis diagnosis, are often the first to detect important clues in their child's course. ${ }^{12-14}$ Patients like Serena Williams, who correctly suspected a postpartum pulmonary embolism but was initially unheeded by her care team, may be the first to know something is wrong. ${ }^{15}$
In this issue of BMJ Quality \& Safety, Fisher and colleagues ${ }^{16}$ studied patients' speaking up to enable organisational learning. Adding a new question to the Hospital Consumer Assessment of Healthcare Providers and Systems survey administered in eight hospitals in Maryland and Washington DC, they asked patients: 'How often did you feel comfortable speaking up if you had any problems in your care?' Half of the respondents reported experiencing problems in their care; they were more likely to be older, more educated and had poorer self-reported overall health. Among these patients, 30\% were not always comfortable speaking up about the problems they experienced. These respondents were more likely to be older, non-English-speaking, admitted through the emergency room, and report poor overall and mental health.

While there is growing awareness of speaking up as a component of safety culture, ${ }^{17-20}$ the research has largely focused on clinicians. If we want to empower patients and families to speak up, we need to ask new questions about hospital culture. Fisher and colleagues ${ }^{16}$ urge us to take the first step.

When we consider speaking up, we need greater granularity. Speaking up about confusing medications or care instructions is not the same as recognising and communicating about patient deterioration, calling out a perceived medical error or speaking up about disrespectful treatment-each distinct problems, with different barriers and motivators. ${ }^{21}$ The proportion of patients $(70 \%)$ in Fisher and colleagues, ${ }^{16}$ study who always felt comfortable speaking up was unexpectedly high, standing in contrast to other reports. ${ }^{14} 2122$ This may reflect the broad potential interpretation of 'problems in care'. We are left to wonder about important details that 
Table 1 Different forms of speaking up: comparison of retrospective patient reports and prospective stopping the line

\begin{tabular}{|c|c|c|}
\hline Characteristics & Patient feedback or reports: after event & Stopping the Line: during event \\
\hline Who patients speak to & $\begin{array}{l}\text { Patient relations, research staff, other non-care-delivering } \\
\text { personnel or online mechanism. }\end{array}$ & Usually clinicians, on whom the patient/family still relies for care. \\
\hline Nature of relationship & Non-binding. & Binding. \\
\hline Nature of reporting & $\begin{array}{l}\text { Neutral; describe event to a neutral or even supportive staff } \\
\text { member. }\end{array}$ & $\begin{array}{l}\text { Personal; raise concerns about a clinician's care to the clinician or } \\
\text { a team member. }\end{array}$ \\
\hline Patient/family risk & Low; especially if invited/recruited to share feedback. & $\begin{array}{l}\text { High; fear of being labelled as a troublemaker or other negative } \\
\text { consequences. }\end{array}$ \\
\hline $\begin{array}{l}\text { Threshold for sharing } \\
\text { information }\end{array}$ & Low; broader range of information more likely to be shared. & $\begin{array}{l}\text { High; patients/families may only take the risk if they perceive dire } \\
\text { circumstances. }\end{array}$ \\
\hline Perceived receptiveness & $\begin{array}{l}\text { High; in some settings feedback is solicited or the receiver's } \\
\text { role is specifically to hear feedback. }\end{array}$ & $\begin{array}{l}\text { Potentially low; clinicians may appear very busy and patients may } \\
\text { fear distracting them. }\end{array}$ \\
\hline Timing & Retrospective; after event has occurred. & Prospective/immediate; before or while event occurs. \\
\hline Goal of communication & $\begin{array}{l}\text { Post-hoc organisational learning; prevent recurrences from } \\
\text { harming others. }\end{array}$ & $\begin{array}{l}\text { Pre-emptive effort to prevent harm from reaching an individual } \\
\text { patient; additional opportunity for organisational learning to } \\
\text { prevent recurrences for others. }\end{array}$ \\
\hline $\begin{array}{l}\text { How patients speak up; } \\
\text { organisational structure }\end{array}$ & $\begin{array}{l}\text { Existing mechanisms such as 'Patient Complaints', often } \\
\text { processed through Patient Relations (but not uniformly } \\
\text { assessed through quality departments). }\end{array}$ & $\begin{array}{l}\text { Few existing mechanisms include patient-activated and family- } \\
\text { activated rapid response systems, present in some organisations. } \\
\text { Most day-to-day care settings lack a clear structure for patients } \\
\text { and families to stop the line. }\end{array}$ \\
\hline
\end{tabular}

would undoubtedly affect patients' comfort speaking up, including the number and nature of the problems in care, and to whom and in what way the patients spoke up (or did not).

We know patients have variable comfort with different types of safety-related behaviours. ${ }^{1423}$ While roughly $70 \%$ of patients and families in the intensive care unit felt very comfortable asking about medications, the percentage dropped to $50 \%$ for speaking up about a possible mistake and 30\% for discussing mismatches in care goals with clinicians. ${ }^{14}$ What patients are speaking up about matters. Similar differences may arise if we also query to whom, when, where and how patients speak up (table 1). Speaking up retrospectively to a third party is conceptually different from speaking up to the care team, on whom the patient still relies, in real time, to help prevent harm. The latter, stopping the line, likely requires greater psychological safety. Increased clarity about these various forms of speaking up can help forge sensible solutions.

We can no doubt learn from all kinds of patient/ family feedback about a broad range of 'problems in care'; here we narrow the focus to stopping the line or speaking up in the moment. In our own research experience over the last decade and in the stories of harmed patients and families, there is a biting truth: many sense a problem, yet find it challenging to speak up or be heard. ${ }^{24} 25$ Creating environments in which patients and families can effectively stop the line will require at least three related changes: (1) changing the research, (2) changing the listening and (3) changing the norms.

\section{CHANGE THE RESEARCH}

Sceptics of patient/family speaking up point to equivocal safety data. While some studies, including those involving patient-initiated and family-initiated rapid response systems, suggest a potential safety benefit, ${ }^{6} 12$ others lack definitive evidence. ${ }^{26}$ But are we using the right yardsticks? Rates of deep vein thrombosis, central line associated blood stream infection or falls, for example, may not give us the full picture. ${ }^{26}$ In a UK study asking patients to report safety concerns, a stunning $86 \%$ of patients did their part. ${ }^{26}$ However, few clinicians showed up to review patient reports and implement safety improvements, and unsurprisingly the study found no difference in safety outcomes.

Fisher and colleagues, ${ }^{16}$ study invites us to reflect more on 'why' speaking up matters, broadening our focus from safety outcomes to the potential for organisations to learn, echoing other studies. ${ }^{62728}$ Intriguingly, in Fisher and colleagues ${ }^{16}$ study, patients who reported problems in their care and always being comfortable to speak up about them provided ratings of patient experience on par with respondents who reported no problems in their care. A link between speaking up and patient experience may generate greater urgency among hospitals as they weigh competing priorities. Research might also pivot to consider other potential beneficial outcomes of speaking up, such as preventing emotional harm ${ }^{29}$ and promoting respect for patients (figure 1). Could support for speaking up help alleviate psychological distress for families like Titcombe's, who may replay events again and again wondering whether there is anything else they could have done? ${ }^{12}$ Or unburden those carrying the guilt of 'you failed your family, you failed yourself...? ? ${ }^{30}$ As we learn that such emotional/psychological harms can last for years and have serious health implications, ${ }^{31}$ comments like these should echo loudly in our ears.

As Berwick ${ }^{32}$ encourages us to consider the 'moral era' in medicine, we will need to become ever more 


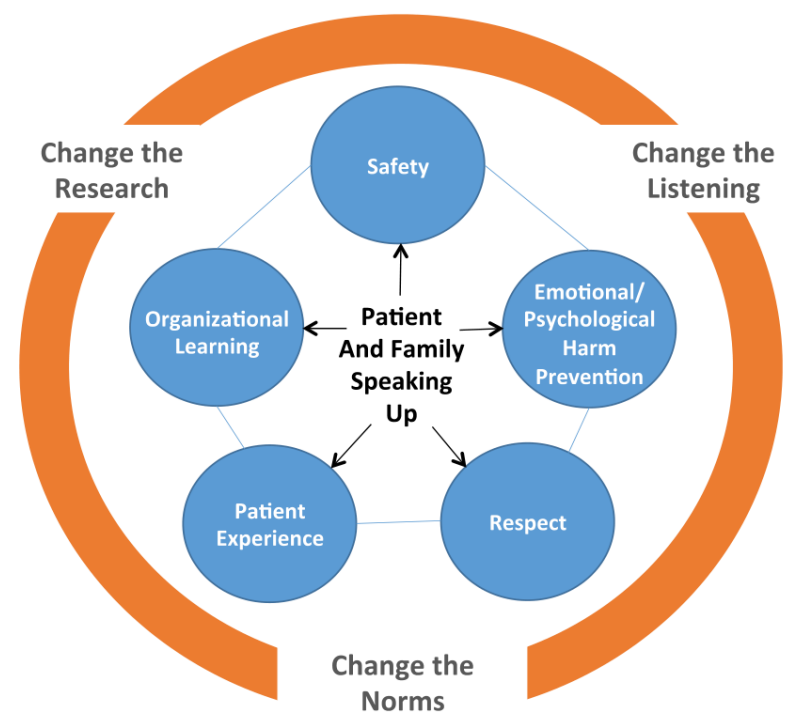

Figure 1 Potential benefits and steps to support patient and family speaking up.

mindful to curate measurements that are meaningful to patients and clinicians, ${ }^{33}$ perhaps using novel patient-reported outcome measures for safety (or unsafety). We should also consider practices that should shift on a moral basis alone. For example, there is intrinsic value in respecting patients by making room for their objections, concerns, fears and knowledge. Organisations like the Joint Commission have developed speaking up initiatives for patients, ${ }^{34}$ and the National Consumer Voice has organised family councils and other resources to unite family voices in order to more effectively communicate concerns to long-term care facilities. ${ }^{35}$ Many harmed patients and families, too numerous to name, have been advocating such change for years. 'Stopping the line', when needed, is what we would want for ourselves and for our loved ones.

\section{CHANGE THE LISTENING}

Studies identify clinician support as a key factor in encouraging patients to speak up. ${ }^{3637}$ Effective change will require pairing 'Speak Up' initiatives with 'Listen Up' strategies, ${ }^{2038} 39$ which harmed patients and families have also long underscored. Lack of meaningful responses when patients go out on a limb to 'stop the line' will not only disincentivise speaking up, it may cause secondary harm. ${ }^{24} 39$ Additional opportunities include involving Patient and Family Advisory Councils in Stop the Line training for patients and clinicians, and inviting patients and families who speak up to morbidity and mortality conferences and/or to participate in quality improvement (QI) efforts. ${ }^{40}$

We can also do a better job of listening when patients speak up retrospectively, after a safety event. A new approach should start with a more patient-centred taxonomy. The term 'patient complaints', commonly used for patient reports about adverse experiences, is imbued with a sense of burden, perhaps even triviality. Instead, we should use empowering language that underscores agency, inclusivity and unique knowledge, such as patient-reported events. Rather than just responding to individual patients through Patient Relations on a case-by-case basis, several experts now compellingly demonstrate that we should be listening across our organisations through a QI process ${ }^{67}$ to amplify organisational learning. Patient-reported events, ${ }^{67}$ patient-activated and family-activated rapid responses, ${ }^{12}$ prelitigation notices and claims, ${ }^{41}$ and other patient feedback should be aggregated and together analysed across departments. ${ }^{6}$ Taking patient reports seriously and publicising lessons learnt can promote visibility and courage for other patients and families to speak up in the future.

\section{CHANGE THE NORMS}

The last change is the hardest: changing our norms. Creating environments in which patients and families can speak up makes more sense now than ever before, because the underlying principles resonate with other existing broad cultural shifts in medicine, such as transparency, patient engagement, shared decision making, inclusivity and respect. However, when it comes to making speaking up the new normal, we will need a multifaceted approach to tackle several barriers to speaking up: patient-related, clinician-related and organisational.

At the patient level, research is needed to inform educational programmes that help patients to develop the language of speaking up. Programmes should help patients distinguish urgent concerns from routine feedback and develop a clear way to describe urgent concerns, perhaps with an 'ask' statement-what do patients and families need that clinicians can act on? This might include adaptation of the CUS model: (1) I am Concerned, (2) I am Uncomfortable, and (3) I feel unSafe or I am Scared. ${ }^{42}$

To help offset worries about negative reactions to voiced concerns, clinicians and organisations can underscore that patient and families hold unique information and that clinicians want to hear from them. ${ }^{143}$ They should highlight clear instructions about who to speak to and how to notify clinicians about urgent issues. ${ }^{14}$ At the same time, clinicians and organisations must also be careful not to inappropriately shift responsibility for safety to patients and recognise that not all patients wish to be involved. ${ }^{23} 44$ Initiatives should be flexible enough to provide opportunity without obligation.

Clinicians and organisations should strive to better understand, and then prevent, circumstances in which patients feel unsafe-even if, or perhaps specifically because, those circumstances may not be currently represented on our QI dashboards. Here too, research is needed to understand patient perceptions of 
'unsafety' and what clinicians need in order to feasibly avoid 'Failure to Listen (and Rescue)'. Comprehensive strategies will require addressing burn-out and resilience, as well as holding clinicians who disrespectfully dismiss patients' and families' concerns accountable. ${ }^{45}$ Finally, organisations should recognise patients and families who speak up, and celebrate their role as part of the healthcare team and safety culture.

Like other analogies in patient safety-aviation, for example-Stop the Line is imperfect. Planes are grounded in bad weather, patients are not. Still there are important lessons to be learnt from aviation, which has inspired meaningful teamwork innovation in medicine. ${ }^{4647}$ Similarly, defects on the production line may be easier for assembly workers to spot than more ambiguous safety threats are for patients. Not all patients are, or want to be, knowledgeable about safety, although many-who navigate chronic illness or experience rare conditions, for example-develop expertise, and others want to learn. Stop the Line may cause false alarms; it will also save some patients. However, even false alarms may play an important role to relieve patients' psychological distress and contribute to a shared understanding between patients and their care teams. Maximising sensitivity, specificity and impact of Stop the Line, and learning more about patient-and-family-defined harm within the constraints of clinician workflow and limited resources, are work to be done. Organising around a concept like respecting patients and families who need to stop the line can mobilise a national conversation.

Many additional questions and opportunities remain. How do we best support vulnerable patients, including those with mental health challenges (as highlighted by Fisher and colleagues ${ }^{16}$ ) or language barriers? How do we make the best and most visible use of patient feedback? How do we re-engineer clinical environments to support clinician listening? As experts urge engaging patients in the diagnostic process, ${ }^{48}$ speaking up will be required at times to prevent diagnostic errors. Serena Williams was able to advocate for herself, repeatedly, and make a difference. What if all patients were empowered to do the same? We applaud Fisher and colleagues for bringing us one step closer.

\section{CONCLUSION}

Developing systems that enable patients to 'Stop the Line' may improve safety, but may also have other important benefits. Empowering every patient and family to speak up, with clear instructions and a genuine invitation, may get messy and may even 'decrease productivity' before it improves care. Patient and family speaking up will be viewed as meaningful if we hold up the right yardsticks, which may include organisational learning, patient experience, respect and prevention of emotional/psychological distress.

True patient engagement requires fully making room for patients at the table, enabling those who feel unsafe to stop the line. That means changing the speaking up research to focus on patient-centred outcomes; better learning from existing patient feedback; and creating the conditions in which patients feel comfortable bringing their voices into our healthcare system, and clinicians and organisations have the resilience to listen and to act.

Acknowledgements The authors thank Eric J Thomas, MD, MPH, Jason M Etchegaray, PhD, Donald M Berwick, MD, MPP, and Linda Kenney for their thoughtful review of the manuscript.

Funding Dr. Martinez is supported by a grant from NIDDK/ NIH (K23DK106511). The views expressed are those of the authors and do not necessarily represent those of NIH.

Competing interests None declared.

Patient consent Not required.

Provenance and peer review Commissioned; internally peer reviewed.

\section{REFERENCES}

1. Sugimori Y, Kusunoki K, Cho F, et al. Toyota production system and kanban system materialization of just-in-time and respect-for-human system. Int J Prod Res 1977;15:553-64.

2. Lenny J. Stop the line manufacturing and continuous integration. 2009. Available: http://www.webcitation.org/ 72waUtIzyAvailable [Accessed 5 Oct 2018].

3. Martin GP, Finn R. Patients as team members: opportunities, challenges and paradoxes of including patients in multi-professional healthcare teams. Sociol Health Illn 2011;33:1050-65.

4. Batalden M, Batalden P, Margolis P, et al. Coproduction of healthcare service. BMJ Qual Saf 2016;25:509-17.

5. Schwappach DL. Review: engaging patients as vigilant partners in safety: a systematic review. Med Care Res Rev 2010;67:119-48.

6. Gillespie A, Reader TW. Patient-centered insights: using health care complaints to reveal hot spots and blind spots in quality and safety. Milbank Q 2018;96:530-67.

7. Pichert JW, Hickson GB, Moore IN. Using patient complaints to promote patient safety: the Patient Advocacy Reporting System (PARS). In: Henriksen K, Battles JB, Keyes MA, eds. Advances in patient safety: new directions and alternative approaches. Bethesda, MD: Agency for Healthcare Research and Quality (AHRQ), 2008: 421-30.

8. Cooper WO, Guillamondegui O, Hines OJ, et al. Use of unsolicited patient observations to identify surgeons with increased risk for postoperative complications. JAMA Surg 2017;152:522-9.

9. Weissman JS, Schneider EC, Weingart SN, et al. Comparing patient-reported hospital adverse events with medical record review: do patients know something that hospitals do not? Ann Intern Med 2008;149:100-8.

10. Bell SK, Gerard M, Fossa A, et al. A patient feedback reporting tool for opennotes: implications for patientclinician safety and quality partnerships. BMJ Qual Saf 2017;26:312-22.

11. Weingart SN, Pagovich O, Sands DZ, et al. What can hospitalized patients tell us about adverse events? Learning from patient-reported incidents. J Gen Intern Med 2005;20:830-6. 
12. Brady PW, Zix J, Brilli R, et al. Developing and evaluating the success of a family activated medical emergency team: a quality improvement report. BMJ Qual Saf 2015;24:203-11.

13. Titcombe J. Parent-activated medical emergency teams: a parent's perspective. BMJ Qual Saf 2015;24:182-3.

14. Bell SK, Roche SD, Mueller A, et al. Speaking up about care concerns in the ICU: patient and family experiences, attitudes and perceived barriers. BMJ Qual Saf 2018;27:928-36.

15. Salam M, . For Serena Williams, Childbirth Was a Harrowing Ordeal. She's Not Alone. New York Times, 2018.

16 Fisher KA, Smith KM, Gallagher TH, et al. We want to know: patient comfort speaking up about breakdowns in care and patient experience. BMJ Qual Saf 2019;28:190-7.

17. Martinez W, Etchegaray JM, Thomas EJ, et al. 'Speaking up' about patient safety concerns and unprofessional behaviour among residents: validation of two scales. BMJ Qual Saf 2015;24:671-80.

18. Richard A, Pfeiffer Y, Schwappach DDL. Development and psychometric evaluation of the speaking up about patient safety Questionnaire. J Patient Saf 2017:1.

19. Ginsburg L. 'Speaking up' climate: a new domain of culture to measure and explore. BMJ Qual Saf 2015;24:661-3.

20. United States Department of Veterans Affairs. Stop the line for patient safety initiative. 2013. Available: http://www. webcitation.org/72wbm611iAvailable [Accessed 5 Oct 2018].

21. O'Hara JK, Lawton RJ. At a crossroads? Key challenges and future opportunities for patient involvement in patient safety. BMJ Qual Saf 2016;25:565-8.

22. Mazor KM, Smith KM, Fisher KA, et al. Speak up! addressing the paradox plaguing patient-centered care. Ann Intern Med 2016;164:618-9.

23. Davis RE, Sevdalis N, Vincent CA. Patient involvement in patient safety: How willing are patients to participate? BMJ Qual Saf 2011;20:108-14.

24. Southwick FS, Cranley NM, Hallisy JA. A patient-initiated voluntary online survey of adverse medical events: the perspective of 696 injured patients and families. BMJ Qual Saf 2015;24:620-9.

25. Iedema R, Allen S, Britton K, et al. Patients' and family members' views on how clinicians enact and how they should enact incident disclosure: the "100 patient stories" qualitative study. BMJ 2011;343:d4423.

26. Lawton R, O'Hara JK, Sheard L, et al. Can patient involvement improve patient safety? a cluster randomised control trial of the Patient Reporting and Action for a Safe Environment (PRASE) intervention. BMJ Qual Saf 2017;26:622-31.

27. Etchegaray JM, Ottosen MJ, Burress L, et al. Structuring patient and family involvement in medical error event disclosure and analysis. Health Aff 2014;33:46-52.

28. Etchegaray JM, Ottosen MJ, Aigbe A, et al. Patients as partners in learning from unexpected events. Health Serv Res 2016;51 Suppl 3:2600-14.

29. Sokol-Hessner L, Folcarelli PH, Sands KE. Emotional harm from disrespect: the neglected preventable harm. BMJ Qual Saf 2015;24:550-3.

30. Delbanco T, Bell SK. Guilty, afraid, and alone - struggling with medical error. N Engl J Med 2007;357:1682-3.
31. Ottosen MJ, Sedlock EW, Aigbe AO, et al. Long-term impacts faced by patients and families after harmful healthcare events. $J$ Patient Saf 2018.

32. Berwick DM. Era 3 for medicine and health care. JAMA 2016;315:1329-30.

33. Tang PC, Smith MD. Democratization of health care. JAMA 2016;316:1663-4.

34. The Joint Commission. Speak up initiatives. 2018. Available: https://www.jointcommission.org/speakup.aspxAvailable [Accessed 30 Sep 2018].

35. National consumer voice for quality long-term care resources for family members: getting help. 2017. Available: http:// theconsumervoice.org/issues/family/family-member-gettinghelp\#Family\%20CouncilsAvailable [Accessed 23 Oct 2018].

36. Waterman $\mathrm{AD}$, Gallagher TH, Garbutt J, et al. Brief report: hospitalized patients' attitudes about and participation in error prevention. J Gen Intern Med 2006;21:367-70.

37. Davis RE, Jacklin R, Sevdalis N, et al. Patient involvement in patient safety: what factors influence patient participation and engagement? Health Expect 2007;10:259-67.

38. Rance S, McCourt C, Rayment J, et al. Women's safety alerts in maternity care: is speaking up enough? BMJ Qual Saf 2013;22:348-55.

39. Bell SK, Etchegaray JM, Gaufberg E, et al. A multi-stakeholder consensus-driven research agenda for better understanding and supporting the emotional impact of harmful events on patients and families. Jt Comm J Qual Patient Saf 2018;44:424-35.

40. Sedlock EW, Ottosen M, Nether K, et al. Creating a comprehensive, unit-based approach to detecting and preventing harm in the neonatal intensive care unit. Journal of Patient Safety and Risk Management 2018;23:167-75.

41. Phillips RL, Bartholomew LA, Dovey SM, et al. Learning from malpractice claims about negligent, adverse events in primary care in the United States. Qual Saf Health Care 2004;13:121-6.

42. Leonard M, Graham S, Bonacum D. The human factor: the critical importance of effective teamwork and communication in providing safe care. Qual Saf Health Care 2004;13 Suppl 1(suppl 1):i85-i90.

43. Frosch DL, May SG, Rendle KA, et al. Authoritarian physicians and patients' fear of being labeled 'difficult' among key obstacles to shared decision making. Health Aff 2012;31:1030-8.

44. Entwistle VA, Mello MM, Brennan TA. Advising patients about patient safety: current initiatives risk shifting responsibility. $J t$ Comm J Qual Patient Saf 2005;31:483-94.

45. Hickson GB, Pichert JW, Webb LE, et al. A complementary approach to promoting professionalism: identifying, measuring, and addressing unprofessional behaviors. Acad Med 2007;82:1040-8.

46. Helmreich RL. On error management: lessons from aviation. BMJ 2000;320:781-5.

47. Dunn EJ, Mills PD, Neily J, et al. Medical team training: applying crew resource management in the Veterans Health Administration. Jt Comm J Qual Patient Saf 2007;33:317-25.

48. National Academies of Sciences, Engineering, and Medicine. Improving diagnosis in health care. National Academies Press, 2016. 